\title{
Method for the Analysis of Multicomponent Exponential Decay Curves*
}

Donald G. Gardner, $\uparrow$ Radiation and Nucleonics Laboratory, Westinghouse Electric Corporation, East Pittsburgh, Pennsylvania

Jeanne C. Gardner $\ddagger$ and George Laush, Department of Mathematics, University of Pittsburgh, Piltsburgh, Pennsylvania

AND

W. Wayne MeInke, Department of Chemistry, University of Michigan, Ann Arbor, Michigan

(Received March 17, 1959)

\begin{abstract}
A frequently encountered problem in many branches of science involves the resolution of experimental data into a sum of independent exponential curves of the form

$$
f(i)=\sum_{i=1}^{n} N_{i} \exp \left(-\lambda_{i} i\right)
$$

in order to estimate the physically significant parameters $N_{i}$ and $\lambda_{i}$. Such problems arise, for example, in the analysis of multicomponent radioactive decay curves, and in the study of the dielectric properties of certain compounds. This paper is concerned with the numerical evaluation of a mathematical approach to the problem. The approach is based on the inversion of the Laplace integral equation by a method of Fourier transforms. The results of the analysis appear in the form of a frequency spectrum. Each true peak in the spectrum indicates a component, the abscissa value at the center of the peak is the decay constant $\lambda_{i}$, while the height of the peak is directly proportional to $N_{i} / \lambda_{i}$. Results obtained on an IBM 650 computer indicate that the method may possess certain advantages over previous methods of analysis.
\end{abstract}

\section{INTRODUCTION}

$\mathbf{T}$ HERE are several types of problems in science in which experimental observations may best be represented by a linear combination of exponentials of the form

$$
f(t)=\sum_{i=1}^{n} N_{i} \exp \left(-\lambda_{i} t\right)
$$

In these problems the parameters $N_{i}$ and $\lambda_{i}$ have biological or physical significance. Therefore, in fitting a function of this form to the data it is not sufficient that the function merely approximate the data closely, but it is also necessary that the parameters be accurately estimated. In Eq. (1) all the exponentials are assumed to be separate and unrelated, i.e., none of the components are produced as the result of the decay of another component. The problem may be stated as follows: a function $f(t)$ is approximated by experimentally determining an estimate of $f(t)$ at a finite number of values of $t$. From this discontinuous set of data it is desired to obtain $n$ (total number of components), and estimates of the $N_{i}$ 's and the $\lambda_{i}$ 's. The essential difficulties in the solution of this problem are that we are dealing with a series of nonlinear equations, that the data are only approximating the function

* This work originated at the University of Michigan as part of the doctoral thesis of one of the authors (DGG) and was supported there in part by the U. S. Atomic Energy Commission. The work has been extended by another author (JCG) at the University of Pittsburgh in partial fulfillment of the M. S. thesis requirements. Portions of this paper have been described at the 1958 San Francisco, California meeting of the American Chemical Society and at the 1958 Urbana, Illinois meeting of the Association for Computing Machinery.

$\dagger$ Present address: Department of Chemistry, University of Arkansas, Fayetteville, Arkansas.

$\mp$ Present address: Department of Mathematics, University of Arkansas, Fayetteville, Arkansas. $f(t)$ over a finite range in $t$, and that the exponential series possesses strongly nonorthogonal properties.

With respect to this problem, the authors have been concerned principally with the analysis of multicomponent radioactive decay curves. However, similar problems arise in the study of (a) first-order chemical kinetics, (b) certain diffusion problems such as neutrons in a moderator, (c) some order-disorder transitions in solid state physics, (d) dielectric properties of certain compounds, (e) relaxation properties of organic polymers, $(\mathrm{f})$ pulses in electrical networks, ( $\mathrm{g}$ ) survival and mortality experiments in the biological sciences, and (h) servo-problems of the guided missile type. Since lately in many cases it has become technically feasible and even convenient to obtain experimental data of reasonably good accuracy, the method of analysis of these data assumes greater importance. The purpose here is to describe a mathematical method of analysis which appears to possess certain advantages over previous methods. The method has been evaluated on the IBM 650 computer located at the University of Pittsburgh.

\section{PREVIOUS METHODS}

By far the most common method used to resolve a decay curve into its components is the graphical approach. Here the data are plotted on semilog paper, and the curve resolved by a repeated subtraction of straight lines. The limitations of the method are apparent and need not be enumerated here. The method is certainly the easiest to perform. The method may be considerably refined by employing a least-squares technique to fit the straight lines, and some error estimation becomes available. The difficulties inherent in the subtraction procedure, however, still remain. Mathematical approaches to this problem have been 
suggested by Prony, ${ }^{1}$ Hudson, ${ }^{2}$ Householder,${ }^{3}$ Cornell, ${ }^{4}$ and Ziegler. ${ }^{5}$ To the authors' knowledge none of these methods, except perhaps that due to Ziegler, are in any sort of routine use. In none of these methods does the number of components "fall out" of the analysis, although in two cases ${ }^{3,5}$ tests are included to determine the number needed to adequately fit the data.

As Lanczos ${ }^{6}$ has pointed out, there are a number of simple and straightforward mathematical solutions to the problem of separating exponentials, but unfortunately enormous practical problems arise when they are applied to experimental data from physical experiments. The principal reason for this is the exceedingly nonorthogonal behavior of the exponential functions. The end result is that the initial data must be extremely accurate if more than two or three exponentials are to be separated. In most cases the accuracy required is far beyond that usually available. In Lanczos' opinion no amount of least-square or other statistical treatment can make up for the extreme sensitivity of the parameters to very small changes in the initial data. In the method described here, a transformation of the initial function to the complex plane is made, wherein the new function exhibits entirely different properties. The hope that this method may succeed where other methods, which deal with purely real numbers, fail is based on the presence of periodic functions which arise during the analysis.

\section{SOLUTION BY FOURIER TRANSFORMS}

The function $f(t)$ in Eq. (1) is in the form of a Dirichlet series which may be expressed as a Stieltjes integral,

$$
f(t)=\sum_{i=1}^{n} N_{i} \exp \left(-\lambda_{i} t\right)=\int_{0}^{\infty} \exp (-\lambda t) d h(\lambda)
$$

The function $f(t)$ may also be expressed in the form of a Laplace integral equation,

$$
f(t)=\int_{0}^{\infty} \exp (-\lambda t) g(\lambda) d \lambda
$$

Here $h(\lambda)$ is a step function and $g(\lambda)$ is a sum of delta functions. Owing to the error inherent in the experimental estimate of $f(t)$ and in the numerical computations necessary to obtain $g(\lambda)$, a plot of $g(\lambda)$ vs $\lambda$ appears in the form of a frequency spectrum. The presence of a peak in the spectrum indicates a component, the abscissa value at the center of a peak is the decay constant $\lambda_{i}$, while the height of the peak is proportional to the coefficient $N_{i}$. The problem then is

${ }^{1}$ F. B. Hildebrand, Introduction to Numerical Analysis (McGraw-Hill Book Company, Inc., New York, 1956).

${ }^{2}$ G. E. Hudson, Am. J. Phys. 21, 362 (1953).

${ }^{3}$ A. S. Householder, U.S. Atomic Energy Commission Rept. ORNL-455 (February, 1950).

4 R. G. Cornell, U.S. Atomic Energy Commission Rept. ORNL2120 (September, 1956).

${ }^{5}$ Keepin, Wimett, and Ziegler, J. Nuclear Energy 6, 1 (1957). ${ }^{6}$ C. Lanczos, A pplied Analysis (Prentice-Hall, Inc., New York, 1956). to determine $g(\lambda)$ given the experimentally determined function $f(l)$. The method advocated here is based on a well-known general approach for solving linear integral equations. ${ }^{7}$ We apply this approach to the specific case of the Laplace integral equation in a manner after Perlis. ${ }^{8}$ A somewhat similar treatment has been described by Paley and Wiener. ${ }^{9}$

We begin with Eq. (3),

$$
f(t)=\int_{0}^{\infty} \exp (-\lambda t) g(\lambda) d \lambda
$$

and proceed to transform the variables $\lambda$ and $t$. Let $\lambda=e^{-y}$ and $t=e^{x}$. Then

$$
f\left(e^{x}\right)=\int_{-\infty}^{\infty} \exp \left[-e^{(x-y)}\right] g\left(e^{-y}\right) e^{-y} d y .
$$

Multiply both sides by $e^{x}$,

$$
e^{x} f\left(e^{x}\right)=\int_{-\infty}^{\infty} \exp \left[-e^{(x-y)}\right] e^{(x-y)} g\left(e^{-y}\right) d y .
$$

Now

$$
F(\mu)=\left[1 /(2 \pi)^{\frac{1}{2}}\right] \int_{-\infty}^{\infty} e^{x} f\left(e^{x}\right) e^{i \mu x} d x,
$$

where $F(\mu)$ is the Fourier transform of $e^{x} f\left(e^{x}\right)$. By combining Eqs. (5) and (6) we obtain

$$
\begin{array}{r}
F(\mu)=\left[1 /(2 \pi)^{\frac{1}{2}}\right] \int_{-\infty}^{\infty}\left\{\int_{-\infty}^{\infty} \exp \left[-e^{(x-y)}\right] e^{(x-y)} g\left(e^{-y}\right) d y\right\} \\
\cdot \exp (i \mu x) d x .
\end{array}
$$

Let $s=x-y$, or $x=s+y$. Then

$$
\begin{array}{r}
F(\mu)=\left[1 /(2 \pi)^{\frac{1}{2}}\right] \int_{-\infty}^{\infty}\left\{\int_{-\infty}^{\infty} \exp \left(-e^{s}\right) e^{s} g\left(e^{-y}\right) d y\right\} \\
\cdot \exp [i \mu(s+y)] d s .
\end{array}
$$

By rearranging terms we have

$$
\begin{aligned}
& F(\mu)=\left[1 /(2 \pi)^{\frac{1}{2}}\right] \int_{-\infty}^{\infty} g\left(e^{-y}\right) \exp (i \mu y) d y \int_{-\infty}^{\infty} \\
& \cdot \exp \left(-e^{s}\right) e^{s} \exp (i \mu s) d s .
\end{aligned}
$$

It is important to keep in mind that the term $e^{s}=e^{x-y}$ is formed by combining in Eq. (5) the term $e^{-y}$, obtained by differentiating $\lambda=e^{-y}$, with $e^{x}$. The kernel $\exp \left[-e^{(x-y)}\right] e^{(x-y)}$ in Eq. (5) is kept intact and later separated from the function $g\left(e^{-u}\right) d y$. This last function is related to the original variables as follows:

$$
g\left(e^{-y}\right) d y=g(\lambda) / \lambda d \lambda .
$$

Hence, when we eventually obtain $g\left(e^{-y}\right)$ as a function

${ }^{7}$ E. C. Titchmarsh, Introduction to the Theory of Fourier Integrals (Oxford University Press, New York, 1937).

${ }^{8}$ A. J. Perlis, U.S. Atomic Energy Commission Rept. NP-786 (September, 1948).

9 R. E. Paley and N. Wiener, Fourier Transforms in the Complex Domain (American Mathematical Society, 1934). 
of $y$ from Eq. (9), this is equivalent to a plot of $g(\lambda) / \lambda$ as a function of $\lambda$.

By returning to Eq. (9) we see that the right-hand side is the product of the Fourier transform $G(\mu)$ of $g\left(e^{-y}\right)$ and the Fourier transform $K(\mu)$ of $\exp \left(-e^{s}\right) e^{s}$. Therefore,

and

$$
F(\mu)=(2 \pi)^{\mathfrak{2}} G(\mu) K(\mu),
$$

$$
G(\mu)=\left[1 /(2 \pi)^{\frac{1}{2}}\right][F(\mu) / K(\mu)] .
$$

By taking the inverse Fourier transform of $G(\mu)$ we obtain

$$
g\left(e^{-y}\right)=(1 / 2 \pi) \int_{-\infty}^{\infty}[F(\mu) / K(\mu)] e^{-i y \mu} d \mu
$$

In this case $K(\mu)$ can be evaluated analytically, and it turns out to be the Euler integral for the complex Gamma function,

$$
K(\mu)=\left[1 /(2 \pi) \frac{1}{3}\right] \Gamma(1+i \mu) .
$$

\section{A FEW DETAILS ON THE NUMERICAL SOLUTION}

Briefly, the method of solution is shown to consist of essentially only two integrations. First, the Fourier transform $F(\mu)$ is found by using Eq. (6). This is divided by the complex Gamma function given in Eq. (14). Finally, $g\left(e^{-y}\right)$ as a function of $y$ is found by using the inverse Fourier transform shown in Eq. (13). From the latter, a plot of $g(\lambda) / \lambda$ vs $\lambda$ may be obtained immediately. Since it is convenient to use equidistant values of $\mu$ in determining $F(\mu), K(\mu)$ can be found most easily from tabulations. ${ }^{10}$ The function $g\left(e^{-u}\right)$ may be found by using the same $\mu$ values or an equidistant subset thereof. Two additional subjects warrant comment: the cutoff error and the setup of the numerical integrations.

\section{A. Cutoff Error}

It is clear that in Eqs. (6) and (13) one cannot numerically integrate from $-\infty$ to $\infty$. Consider Eq. (6). Here we must introduce the limits $\pm x_{0}$ which are the cutoff points of the integral. Instead of Eq. (6) we have

$$
(2 \pi)^{\frac{1}{2}} F(\mu)=\int_{-x_{0}}^{x_{0}} e^{x} f\left(e^{x}\right) \exp (i \mu x) d x+E\left(x_{0}, \mu\right) .
$$

The calculated $(2 \pi)^{\frac{1}{2}} F(\mu)$ is in error by at least the amount $E\left(x_{0}, \mu\right)$. The major difficulty of the method is now apparent. We are trying to simulate a curve with an abrupt cutoff at $x_{0}$ by a sum of exponentials that extend to $x=\infty$. This has the effect of adding into $F(\mu)$ Fourier components which extend the range in $\mu$ on which $F(\mu)$ maintains appreciable value. Since $K(\mu)$ diminishes rapidly with increasing $\mu$, for some value of $\mu$ the quotient $F(\mu) / K(\mu)$ in Eq. (13) begins

\footnotetext{
${ }^{10}$ Natl. Bur. Standards, Appl. Math. Ser. 34 (1954), "Tables of the gamma function for complex arguments."
}

to grow without bound. The end result is found to be error ripples in the plot of $g\left(e^{-y}\right)$ vs $y$ which tend to obscure the results. Hence, it is necessary that a finite $x_{0}$ exist such that the value of $E\left(x_{0}, \mu\right)$ is sufficiently small that a good solution is possible. It is most unfortunate that once $F(\mu)$ has been warped by the cutoff, the warping cannot be removed by any subsequent mathematical treatment.

If it is not possible to experimentally follow the decay curve for a long enough time, the data must be treated in some appropriate manner. For example, the longestlived component might be extrapolated to give the necessary information. Even a somewhat inaccurate extrapolation usually yields far better results than if no extrapolation is made. Alternatively, it might be possible to subtract off the longest-lived component before the analysis. Finally, a drastic and unrecommended step is to introduce a convergence factor into Eq. (13).

The cutoff errors in Eq. (6) tend to increase the height of the error ripples in the final results. A cutoff at $\mu= \pm \mu_{0}$ in Eq. (13), on the other hand, has been shown to primarily affect the frequency of the error ripplies and the breadth of the true peaks. The larger the value of $\mu_{0}$, the narrower and more-well-defined are the component peaks. The maximum useable value of $\mu_{0}$ depends on how good the initial data are and on the cutoff at $x_{0}$. If $\mu_{0}$ is chosen too large, the cutoff at $x_{0}$ causes the amplitude of the error ripples to increase. If $\mu_{0}$ is chosen too small, there is an unnecessary loss in resolution of the peaks in the final result. The greater the value in $\mu$ at which $F(\mu) / K(\mu)$ remains well behaved, the finer the resolution can be made in the final results.

\section{B. Numerical Integration}

It is convenient to adjust the units of $t$ in the initial data such that the decay constants $\lambda_{i}$ fall in the range from 0 to 1 . In other words, the half-life of the shortestlived component should not be less than 0.693 units of $t$. This is no restriction since the range of $t$ is infinite and the scale with which $t$ is measured can be arbitrary. Next, each value of $f(l)$ is multiplied by its value of $t$ and the results plotted as $e^{x} f\left(e^{x}\right)$ vs $x$. $(2 \pi)^{\frac{1}{3}} F(\mu)$ is then related to the area under this new curve.

Whereas $t$ ranges in principle from 0 to $\infty, x$ now ranges from $-\infty$ to $\infty$. Hence, we can set up the integral

$$
F(\mu)=\left[1 /(2 \pi)^{\frac{1}{2}}\right] \int_{0}^{x_{0}}\left[f^{*}(x)+f^{*}(-x)\right] e^{i \mu x} d x,
$$

or more conveniently,

$$
\begin{array}{r}
F(\mu)=\left[1 /(2 \pi)^{\frac{1}{2}}\right] \int_{0}^{x_{0}}\left\{\left[f^{*}(x)+f^{*}(-x)\right] \cos \mu x\right. \\
\left.+i\left[f^{*}(x)-f^{*}(-x)\right] \sin \mu x\right\} d x .
\end{array}
$$


Here we define $f^{*}(x) \equiv e^{x} f\left(e^{x}\right)$. This yields real and imaginary parts of $F(\mu)$ which we term $F_{c}$ and $F_{s}$, respectively. $K(\mu)$ is similarly composed of real and imaginary parts $K_{c}$ and $K_{\mathrm{s}}$. Hence

$(2 \pi)^{\frac{t}{3}} G(\mu)=\frac{F(\mu)}{K(\mu)}=\frac{F_{c}+i F_{s}}{K_{c}+i K_{s}}=\frac{\left(F_{c}+i F_{s}\right)\left(K_{c}-i K_{s}\right)}{K_{c}^{2}+K_{s}^{2}}$.

Next

$$
\begin{array}{r}
\frac{1}{2 \pi} \int_{-\mu_{0}}^{\mu_{0}} \frac{F(\mu)}{K(\mu)} e^{-i y \mu} d \mu=\frac{1}{2 \pi} \int_{-\mu_{0}}^{\mu_{0}} \frac{\left(F_{c}+i F_{s}\right)\left(K_{c}-i K_{s}\right)}{K_{c}{ }^{2}+K_{s}{ }^{2}} \\
\cdot(\cos y \mu-i \sin y \mu) d \mu .
\end{array}
$$

All odd terms vanish in Eq. (19) yielding

$$
\begin{aligned}
& g\left(e^{-y}\right) \\
& =\frac{1}{\pi} \int_{0}^{\mu_{0}}\left(\frac{F_{c} K_{c}+F_{s} K_{s}}{K_{\mathrm{c}}{ }^{2}+K_{s}{ }^{2}} \cos y \mu+\frac{F_{s} K_{c}-F_{c} K_{s}}{K_{c}{ }^{2}+K_{s}{ }^{2}} \sin y \mu\right) d \mu .
\end{aligned}
$$

If $F(\mu)$ is determined for an equidistant set of $\mu$ 's ranging from 0 to $\mu_{0}$ in steps of say $\Delta \mu=0.1$, it is then convenient to use tabulated values of the gamma function..$^{10}$ In most cases it suffices to use a simple numerical integration scheme such as Simpson's Rule for Eqs. (17) and (20), although if the accuracy of the initial data warrants it, a more refined procedure such as that of Filon ${ }^{11}$ may be employed. Normally, it is not convenient to take experimental data in precisely the right intervals that best suit the method of analysis to be used to evaluate the data. In the present case, two alternatives arise. Either an unequal interval integration scheme can be employed to obtain the Fourier transform function $F(\mu)$, or an interpolation procedure might be used to obtain functional values of the initial data at the desired intervals. If a large number of data are available one might choose the former approach, whereas if the initial data are available at only a few points or if the data are badly scattered the latter method might prove the more desirable.

\section{RESULTS OF THE NUMERICAL EVALUATION}

To test out the method, we have constructed one-, two-, three-, and four-component decay curves. In the most accurate of these curves $\left[f(t)=100 e^{-0.02 t}\right]$ the data ranges in accuracy from about 1 part in $10^{5}$ at the beginning of the curve to 3 parts in 10 at the end. The accuracy of the remaining curves ranges from about 0.5 to 1 part in $10^{4}$ at the beginning to perhaps 5 parts in 10 at the end, except for the curves wherein the data are deliberately distorted in order to study a particularly desired effect. To simplify the calculations the data are constructed at equal intervals in the logarithm of $t$. This yields many points near the be-

${ }^{11}$ L. N. G. Filon, Proc. Roy. Soc. Edinburgh 49, 38 (1928111 L.
TABLE I. Input data for the accurate decay curve $f(t)=$ $100 e^{-0.02 t}$, together with $\%$-deviation factors used to simulate a decay curve with scatter.

\begin{tabular}{cccc}
\hline \multicolumn{1}{c}{$t$} & $x$ & $f(t)$ & \% deviation \\
\hline 0 & $-\infty$ & 100.000 & $\ldots$ \\
1.0000 & 0 & 98.020 & +4.1 \\
1.2840 & 0.25 & 97.467 & -8.0 \\
1.6480 & 0.50 & 96.758 & -6.9 \\
2.1170 & 0.75 & 95.854 & -6.4 \\
2.7183 & 1.00 & 94.706 & -1.9 \\
3.4903 & 1.25 & 93.259 & 0 \\
4.4817 & 1.50 & 91.426 & +0.5 \\
5.7546 & 1.75 & 89.128 & -1.8 \\
7.3891 & 2.00 & 86.261 & +3.6 \\
9.4877 & 2.25 & 82.717 & -2.5 \\
12.182 & 2.50 & 78.380 & +0.7 \\
15.643 & 2.75 & 73.134 & -2.1 \\
20.086 & 3.00 & 66.915 & +2.8 \\
25.790 & 3.25 & 59.703 & 0 \\
33.115 & 3.50 & 51.567 & +1.9 \\
42.521 & 3.75 & 42.724 & -1.2 \\
54.598 & 4.00 & 33.556 & -1.9 \\
70.105 & 4.25 & 24.608 & -3.6 \\
90.017 & 4.50 & 16.524 & -1.5 \\
115.584 & 4.75 & 9.909 & 0 \\
148.413 & 5.00 & 5.139 & -1.4 \\
190.566 & 5.25 & 2.212 & +4.0 \\
244.692 & 5.50 & 0.7493 & +5.5 \\
314.191 & 5.75 & 0.1866 & 0 \\
403.429 & 6.00 & 0.0313 & -15.9 \\
518.013 & 6.25 & 0.0032 & +122.0 \\
665.42 & 6.50 & 0 & 0 \\
\hline
\end{tabular}

a Data points between $t=0$ and $t=1$ not listed.

ginning of the decay curve where $f(t)$ is decreasing rapidly, and relatively few points at widely spaced intervals in $t$ near the end of the curve where the longest-lived component is decaying slowly. The data thus produced are more realistically distributed with respect to the variable $t$ than would have been the case had equal intervals in $t$ been chosen, since one normally tends to take more experimental points where the data are changing rapidly. An example of the decay curve data is given in Table I. Here the initial data for the single component curve $f(t)=$ $100 e^{-0.02 t}$ are shown together with the factors used to distort the data when the effect of scatter in the original data is studied (see Sec. V.D). The points between $t=0$ and $t=1$ are omitted from the table since they are normally obtained by interpolation. The accuracy of these points was about 1 part in $10^{5}$ for this curve.

\section{A. Effect of Cutoff with Respect to $\mu$}

By using Eq. (20) we determine a plot of $g\left(e^{-y}\right)$ vs $y$ by integrating the expression from 0 to $\mu_{0}$ for each desired value of $y$. Such a plot is equivalent to a plot of $g(\lambda) / \lambda$ vs $\lambda$. In Fig. 1 we show the effect of increasing the final integration range from $\mu_{0}=2$ to $\mu_{0}=4$, by using the data for the single-component curve where $\lambda=0.02$. It is seen that the principal peak falls in the same place on each curve, just at the proper $\lambda$ value. The breadth of the principal peak and the smaller 


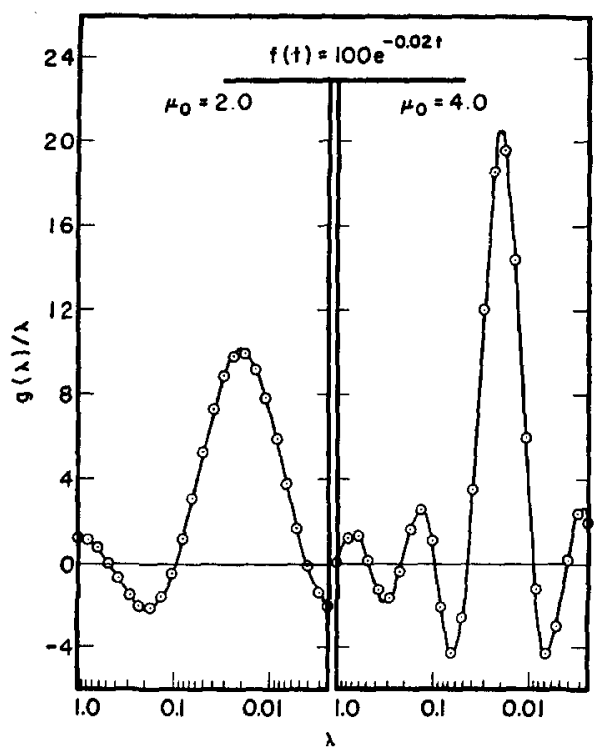

FIG. 1. Effect of increasing $\mu_{0}$ from 2.0 to 4.0 in the analysis of a single-component decay curve. $\lambda=0.02, x_{0}=7.0$. Ordinate units are arbitrary.

ripples appears to be caused by errors, primarily cutoff errors, in the calculation and errors in the initial data. As the range in $\mu$ is extended, the resolution of the peak becomes better. In Fig. 2 the range in $\mu$ is extended to $\mu_{0}=6$ and $\mu_{0}=8$, and again the resolution increases progressively. The positions of the peaks in the error ripple change as a function of $\mu_{0}$, whereas the position of the true peak does not. This fact provides one method for distinguishing small true peaks from error ripples-simply change $\mu_{0}$ and note which peaks do not shift position.

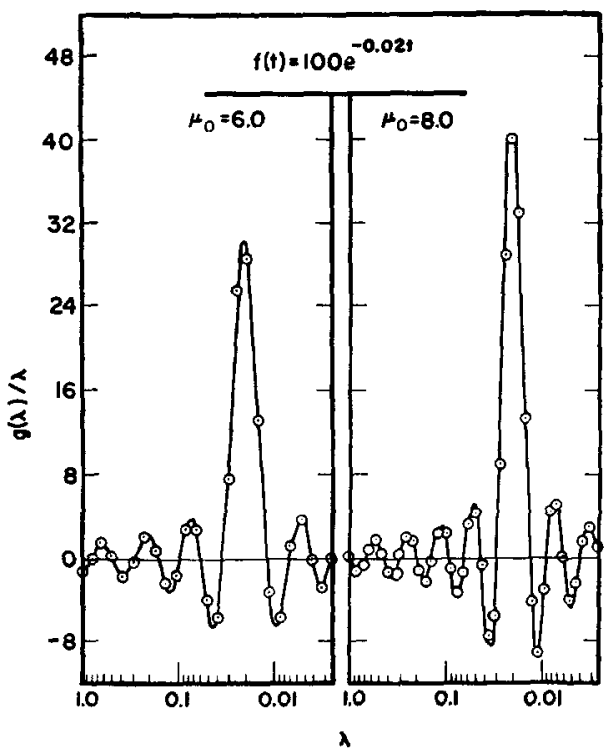

FIG. 2. Effect of increasing $\mu_{0}$ from 6.0 to 8.0 in the analysis of a single-component decay curve. $\lambda=0.02, x_{0}=7.0$. Ordinate units are arbitrary.

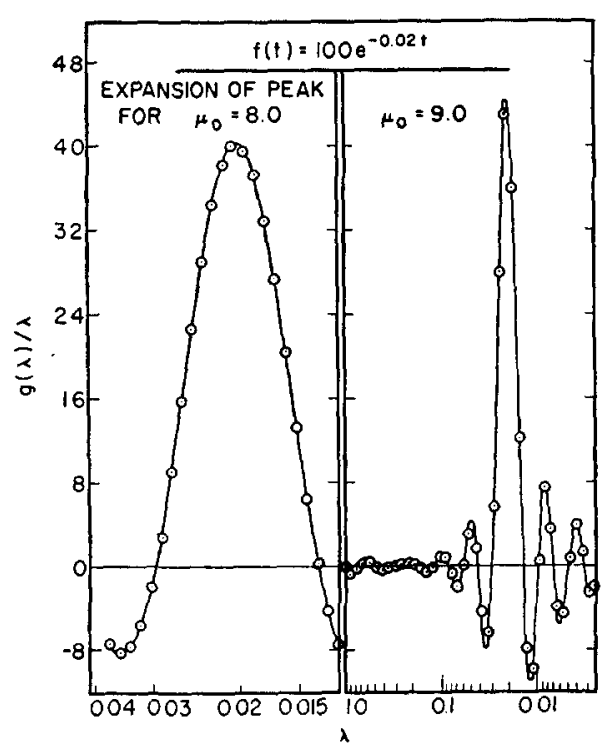

FIG. 3. Detail of the peak shown in Fig. 2 for $\mu_{0}=8.0$, and the results for the analysis of a single-component decay curve with $\mu_{0}=9.0 . \lambda=0.02, x_{0}=7.0$. Ordinate units are arbitrary.

Once the positions of the true peaks are found, they may be examined more closely by taking smaller intervals in $y$. The left-hand curve in Fig. 3 shows an expanded view of the $\mu_{0}=8$ peak of Fig. 2. Also shown in Fig. 3 is the curve obtained for $\mu_{0}=9$. While the resolution is excellent and the center of the peak falls at the proper place, it is seen that the error ripples are no longer symmetrical with respect to the true peak. In order to obtain finer resolution without increasing the height of the error ripples, it is necessary to use better initial data and/or perhaps a more ac-

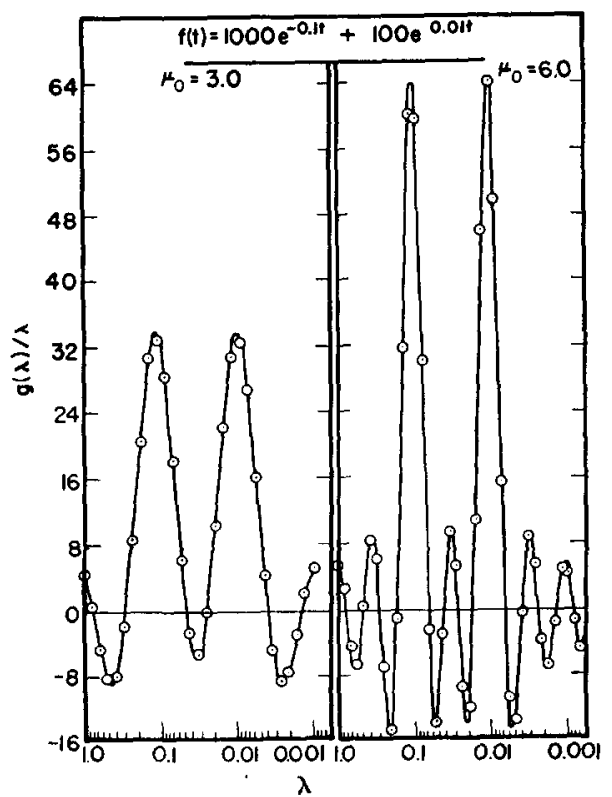

FIG. 4. Effect of increasing $\mu_{0}$ from 3.0 to 6.0 in the analysis of a two-component decay curve. $\lambda_{1}=0.1, \lambda_{2}=0.01, x_{0}=7.0$. Ordinate units are arbitrary. 
curate integration scheme such as the one due to Filon. ${ }^{11}$ Although not checked experimentally, it may only be necessary to extract more points from the initial data by some interpolation procedure so as to be able to extend $\mu_{0}$ to a higher value without introducing more error.

Figure 4 illustrates the case of a two-component curve with $\mu_{0}$ increasing from 3 to 6 . In both curves the principal peaks are of the same height. This is because the coefficient of the second component, which is a factor of 10 smaller than that of the first component, is divided by a $\lambda$ which is also a factor of 10 smaller than the $\lambda$ for the first component. Furthermore, the breadth of each of the two peaks is the same. This means the resolution is constant over the entire range due to the fact that the method treats the initial data as a whole and minimizes the error uniformly over the entire curve. This fact is useful in analyzing unknown curves. For example, if two components have $\lambda$ values so close together that the resultant peaks cannot be completely resolved, the peak representing their sum may be wider than expected for a singlecomponent peak. The symmetry of the peak gives a rough indication of the relative amounts of each component.

In Fig. 5 the results of the analysis of a three-component decay curve are displayed. Again the increase of $\mu_{0}$ from 6 to 8 greatly improves the accuracy of the final results. Finally, in Fig. 6 the results obtained from the analysis of a four-component curve are shown. In this curve, the $\lambda$ values range from 0.5 to 0.01 while the coefficients range from 3750 to 100 . In Table II, the relative height of each component peak $\left[g\left(\lambda_{i}\right) / \lambda_{i}\right]$ and the associated $\lambda_{i}$ value as determined graphically from the data in Fig. 6 are compared with the actual

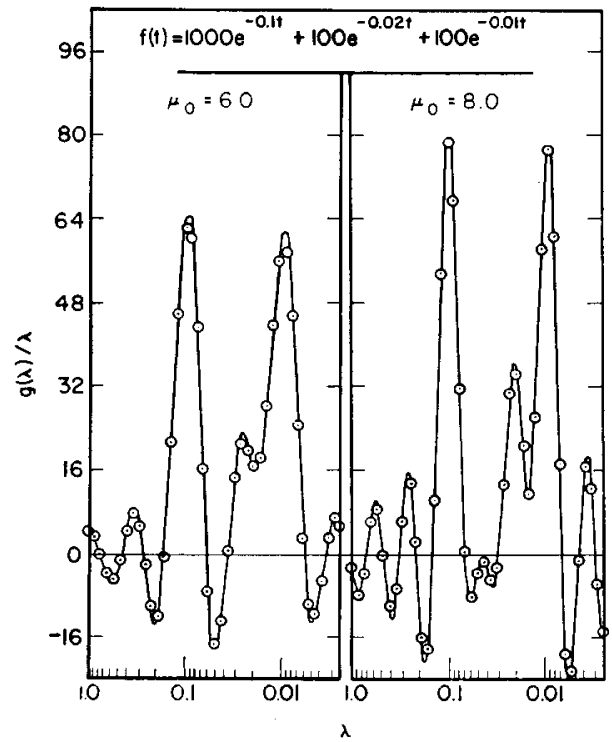

FIG. 5. Effect of increasing $\mu_{0}$ from 6.0 to 8.0 in the analysis of a three-component decay curve. $\lambda_{1}=0.1, \lambda_{2}=0.02, \lambda_{3}=0.01$, $x_{0}=7.0$. Ordinate units are arbitrary.

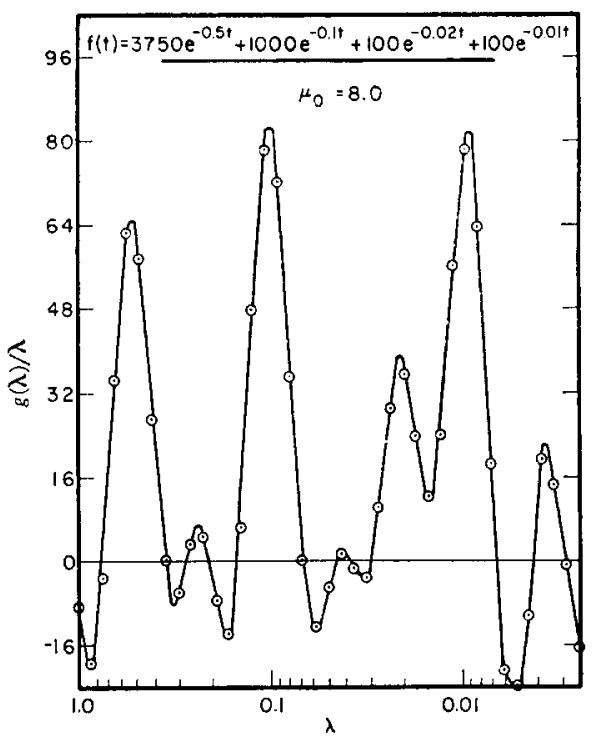

FIG. 6. Results of the analysis of a four-component decay curve $\mu_{0}=8.0 . \lambda_{1}=0.5, \lambda_{2}=0.1, \lambda_{3}=0.02, \lambda_{4}=0.01, x_{0}=7.0$. Ordinate units are arbitrary.

values. The deviations from the true values give an indication of the accuracy of the method. Four components are determined from only 29 points on the gross decay curve in the range from $t=1$ to $t=1100$ together with the associated points in the range from $\mathrm{t}=0$ to $t=1$ which are normally found by interpolation. By using more points in the initial data and by extending the limit $\mu_{0}$ slightly, a more accurate determination of the parameters is possible.

The relatively large-error ripple peak, which occurs at a $\lambda$ value of about 0.0035 , can be distinguished from a true peak by several means. First, the original data can be checked to see if a component with a half-life roughly three times as long as that of the $\lambda=0.01 \mathrm{com}$ ponent is reasonable. Next, the final integration limit $\mu_{0}$ can be changed and the shift in position of the error ripples noted. Finally, the width of the suspected peak at its base [where $g(\lambda) / \lambda=0$ ] can be compared with the width of true peaks, since error ripples always appear narrower than true peaks. The $\sim 5 \%$ shift in the apparent $\lambda$ value of the $\lambda=0.01$ peak is not encountered when a $\lambda=0.02$ and $\lambda=0.01$ two-component curve is analyzed, but does occur in the results of the three-component analysis. Since experience shows that a $5 \%$ shift is relatively large for a major peak, it is possible that the data for the $\lambda=0.1$ decay curve are slightly in error.

The range in $\lambda$ can easily be extended to cover a much greater spread in half-lives provided the coefficients $N_{i}$ tend to decrease as the $\lambda_{i}$ values decrease. What is important here is the relative values of the quotient $N_{i} / \lambda_{i}$. Although in Fig. 6 the $\lambda_{i}$ and the $N_{i}$ values vary by factors of 50 and 37.5 , respectively, the quotients $N_{i} / \lambda_{i}$, i.e., the heights of the peaks, differ at most by a factor of 2 . The maximum ac- 
TABLE II. Comparison of the results graphically determined from Fig. 6 for the four-component decay curve with the actual values. Height of the $\lambda=0.1$ peak normalized to $1.000 . g\left(\lambda_{i}\right) / \lambda_{i}$ is termed $H_{i}$.

\begin{tabular}{lccccccccc}
\hline & $H_{1}$ & $H_{2}$ & $H_{3}$ & $H_{4}$ & $\lambda_{1}$ & $\lambda_{2}$ & $\lambda_{3}$ & $\lambda_{4}$ \\
\hline Actual value & 0.750 & 1.000 & 0.500 & 1.000 & 0.500 & 0.100 & 0.02 & 0.010 \\
Graphical estimates (Fig. 6) & 0.769 & 1.000 & 0.466 & 0.988 & 0.516 & 0.0985 & 0.021 & 0.0095 \\
\% deviation from actual values & +2.5 & 0 & -6.8 & -1.2 & +3.2 & -1.5 & +5.0 & -5.0 \\
\hline
\end{tabular}

ceptable difference in $N_{i} / \lambda_{i}$ depends on the integration limit $\mu_{0}$ and on the separation in $\lambda_{i}$ values. For example, with similar $\lambda_{i}$ values such as 0.02 and 0.01 in a twocomponent curve, a difference in peak heights of 3 or 4 might be the maximum variation which still permits a reasonably accurate determination of the parameters $N_{i}$ and $\lambda_{i}$ for a $\mu_{0}$ of the order of 8 . On the other hand, with widely separated $\lambda_{i}$ values such as 0.5 and 0.02 in a two-component curve, the peak heights might differ by a factor of 10 and still allow a useful solution to be found for a $\mu_{0}$ of the order of 8 . Obviously, the limiting factor is the relative heights of the error ripples compared to the peak heights.

\section{B. Effect of Cutoff with Respect to $X$}

In all cases the experimentally determined function $f(t)$ must be cut off at some finite value of $t$. Actually, under the change of variables, we are interested in the function $e^{x} f\left(e^{x}\right)$ is $x$. Consider the case of the singlecomponent curve with $\lambda=0.02$. In Fig. 7 we show a plot of the function $e^{x} f\left(e^{x}\right)$ and two cases of cutoff. In the first case $\left|x_{0}\right|=5.25$, while $\left|x_{0}\right|=6.25$ in the second case. It should be kept in mind that equal intervals in $x$ correspond to exponentially increasing intervals in $t$. While little information is lost on the left, a considerable amount can be lost on the right. Even with a cutoff at $\left|x_{0}\right|=7$, the amount lost is not negligible for high $\mu_{0}$ values $\left(\mu_{0} \geq 9\right)$ since the curve actually extends to $x= \pm \infty$. Figure 8 shows the results

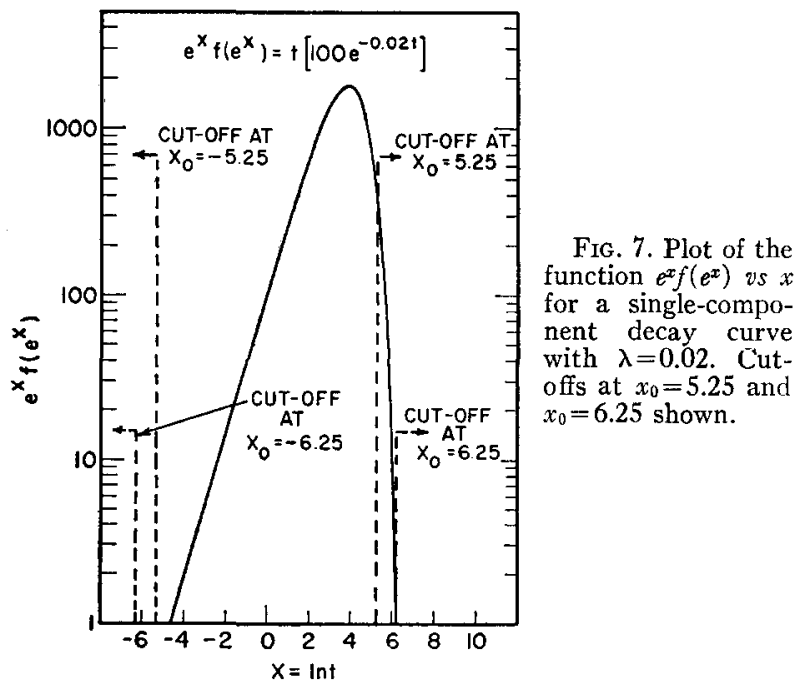

of a cutoff at $\left|x_{0}\right|=5.25$. When the final integration is carried out to $\mu_{0}=6$, the error ripples completely mask the true curve. The dark triangle shows the expected height of the true peak. However, even in this poor case where the initial data are cut off after about 5.5 half-lives, excellent results can be obtained if we are willing to accept poorer resolution. By restricting $\mu_{0}$ to 4 the error ripples are greatly reduced and the true peak appears at the proper $\lambda$ value.

The results for the case of a cutoff at $\left|x_{0}\right|=6.25$ are as follows. For $\mu_{0}=6$ there is no apparent deviation from the results shown in Fig. 2 where the data are cut off at $\left|x_{0}\right|=7$. Only when $\mu_{0}$ is increased to 8 do the error ripples appear larger than they are in Fig. 2. Even in this case the true peak appears at the proper $\lambda$ value.

\section{Effect of Poor Extrapolation of the Initial Data}

Since a large cutoff in the initial data can exhibit such a profound influence on the final results, it is naturally of interest to see if it is advantageous to extrapolate the initial data, even if the extrapolation is somewhat in error. Two decay curves are constructed

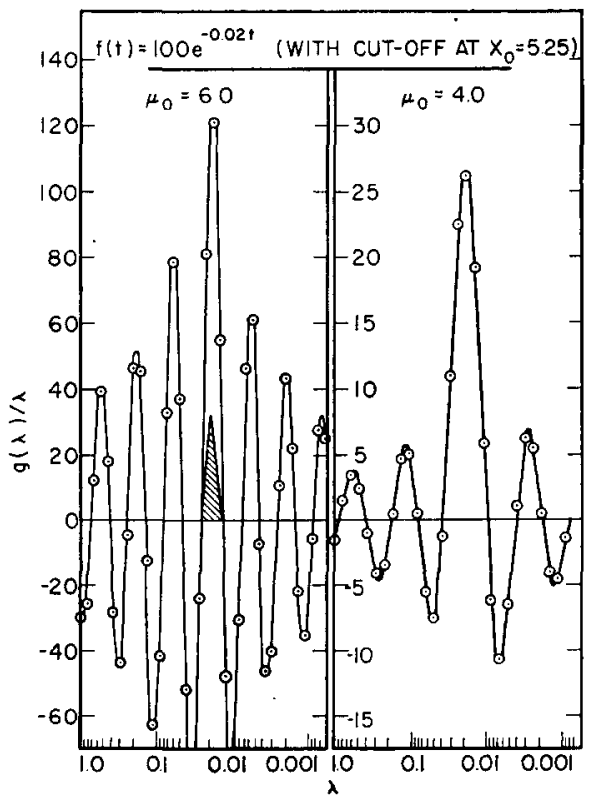

Fig. 8. Effect of cutoff at $x_{0}=5.25$ in the analysis of a singlecomponent decay curve with $\mu_{0}$ decreasing from 6.0 to 4.0 . $\lambda=0.02$. Ordinate units are arbitrary. 
based on the single-component $\lambda=0.02$ data. In each case the accurate data ends at $\left|x_{0}\right|=5(t=148.4)$ where an abrupt change is made to a $\lambda$ value of 0.023 or 0.017 . These curves are called "low extrapolation" and "high extrapolation," respectively. The curves are then extended out to $\left|x_{0}\right|=7$. The final results obtained for each case appear in Fig. 9 and may be compared with Fig. 8 where the initial data are cut off at $\left|x_{0}\right|=5.25$.

In the case of the high extrapolation, the initial data appear to contain an additional component with a $\lambda$ value less than 0.02 . Thus, for $\mu_{0}=6$ we see a double peak near $\lambda=0.02$. The presence of the additional ficticious component has shifted the position of the true peak to a larger value, and has caused large error ripples at the beginning of the curve due to the fact that the initial data in the range from $t=0$ to $t=148.4$ do not contain the additional component. Although badly distorted, the results are still of much more value than if the extrapolation had not been made, as in the case of the cutoff at $\left|x_{0}\right|=5.25$. At a sacrifice in resolution, the high extrapolation data yield excellent results for $a \mu_{0}=4$. In the case of the low extrapolation and $\mu_{0}=6$ we again find that even a poor extrapolation is much better than a large cutoff. With $\mu_{0}=6$ the error ripples are large, but the true peak appears at just about the proper place. By decreasing $\mu_{0}$ much better results can be obtained. It may be said, then, that even a poor extrapolation usually yields better results than if the data are not extrapolated at all, but care must be taken not to try to push the resolution beyond the accuracy inherent in the data. Furthermore, it is safer to err on the low side rather than the high side when extrapolating.

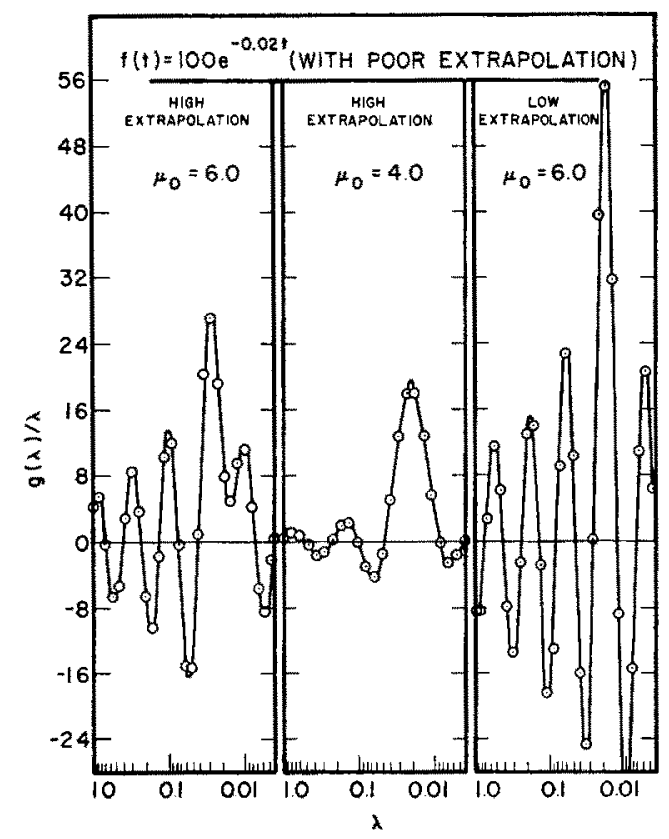

Fig. 9. Effect of poor extrapolation of the initial data in the analysis of a single-component decay curve. $\lambda=0.02, x_{0}=7.0$ Ordinate units are arbitrary.

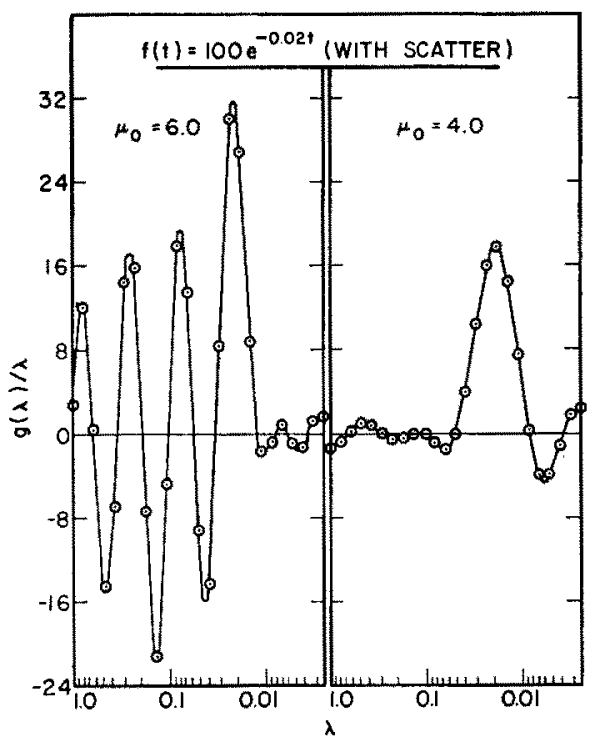

Frg. 10. Effect of scatter in the initial data in the analysis of a single-component decay curve. $\lambda=0.02, x_{3}=7.0$. Ordinate units are arbitrary.

\section{Effect of Scatter in the Initial Data}

Since we are dealing with a Fourier-type analysis we expect the method to exert a smoothing effect, in the least-square sense, on the data. The greater the smoothing effect the more statistical scatter can be tolerated in the initial data. To investigate this effect we have constructed a curve using the $\lambda=0.02$ singlecomponent data which contain a certain amount of more or less random scatter. Table I lists the percent deviation and the sign with which each point of the accurate data is changed to produce the scatter effect. While the scatter so introduced may seem large with respect to certain types of actual experimental data, it is just those cases where accurate data are not available that are in most need of a method of analysis. In Fig. 10 the results obtained with the poor data are shown for $\mu_{0}$ values of 6 and 4 . For $\mu_{0}=6$ large error ripples are produced, but the true peak still appears at the proper $\lambda$ value. By reducing $\mu_{0}$ to 4 relatively excellent results are obtained due to the reduction in the error ripples. It is anticipated that smoothing of the data prior to analysis appreciably improves the final results. It should be possible to successfully analyze decay curves containing considerably more scatter provided that the data do not contain a bias and that a sufficient amount of initial data is available to provide a basis for the smoothing effect.

\section{DISCUSSION}

As mentioned in the introduction, it is hoped that the method described here may be of use in the analysis of data from a wide variety of experimental problems in the physical, chemical, and biological sciences. The ultimate accuracy, of course, depends upon the data available. For example, when analyzing a mixture of 
radioactive isotopes it is usually possible to accumulate a large amount of accurate decay-curve data. This is one case where a high level of resolution in the final results should be possible. When the usual radioactive decay-rate data are analyzed, the heights of the resultant peaks are proportional to the number of atoms of each species.

Another case where good resolution should be possible occurs when the measurements may be repeated as often as desired and the results from each set of measurements averaged. Dielectric relaxation properties, for example, lend themselves to repeated measurements. Certain types of chemical kinetics data, on the other hand, or measurements involving the rates at which injected materials disseminate in a living organism cannot always be obtained with great accuracy. The meager or poor data often represent reactions in which there are only one or two components. Hence, a lower level of resolution may give a satisfactory solution that is not available when using other types of mathematical approaches since the detrimental effects due to the nonorthogonal properties of the exponential series can, to some extent, be avoided by using the present method.

A few remarks of an empirical nature on the subject of errors are applicable here. With reasonable resolution, the function $g\left(e^{-y}\right)$ vs $y$ produces symmetrical peak profiles for true components. Furthermore, the breadth of the true peaks is independent of $y$. Therefore, an error estimate may be obtained from the peaks themselves, and if a peak appears unsymmetrical or wider than another peak, the presence of unresolved components is indicated. The positions of the peaks in the error ripple depend upon $\mu_{0}$, while the positions of the true peaks do not. This fact can be used by merely carrying out the final integration to two different $\mu_{0}$ values and noting which peaks shift position. Also, the width of the base of a true peak is wider than the base width of an error ripple. Another test depends on the regular damping of the amplitudes of the error ripples. A divergence from the regular trend signals the presence of a true peak. Finally, it may be possible to obtain an error estimate for the integration scheme and also for the cutoff error. When there is doubt about a particular component, calculated decay curves can be constructed both with and without the suspected component, and the scatter of the experimental points about the calculated curves can be examined statistically with respect to "goodness of fit."

The fact that the results of an analysis are available as a functional display particularly at several levels of fineness of resolution, rather than merely a set of values for the parameters, is quite advantageous. Although the determination of the number of components in principle is reduced to counting the number of true peaks, in difficult cases it is desirable to view the solution as a whole in reference to the mutual interaction of all of the parameters. A study of the curves representing the final results at several levels of resolution can provide a sounder basis for the application of human judgment in cases where there is only a small amount of one of the components or where two components have similar $\lambda$ values. Another advantage is that the initial data are not required to be as accurate as in other methods, and full use is made of the accuracy that is available since the data are treated as a whole, as opposed to "subtraction-type" methods wherein all but the shortest-lived components are determined by using fewer points than are actually available. Furthermore, the occurrence of half-lives very close together in magnitude does not endanger the entire solution as in other methods.

The major limitation of the method appears to be that information concerning the decay of the longestlived component must be available for many half-lives. This limitation may not be too serious and several methods for treating the data in this regard have been suggested.

\section{ACKNOWLEDGMENT}

The authors are indebted to Professor Alan Perlis of the Carnegie Institute of Technology and Professor John Carr III of the University of North Carolina for many valuable discussions of the problem, and to Professor W. Kehl for making available the University of Pittsburgh's IBM 650 computer. 